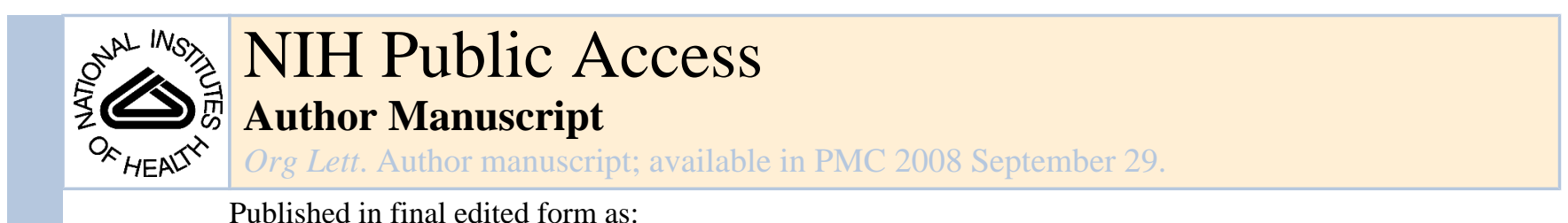

Published in final edited form as:

Org Lett. 2006 December 7; 8(25): 5865-5868. doi:10.1021/o1062470p.

\title{
Thermolysis of Iso-Migrastatin and Its Congeners via [3,3]- Sigmatropic Rearrangement: A New Route to the Synthesis of Migrastatin and Its Analogues
}

\author{
Jianhua Ju${ }^{1}$, Si-Kyu Lim ${ }^{1}$, Hui Jiang ${ }^{1}$, Jeong-Woo Seo ${ }^{1}$, Yeng Her ${ }^{3}$, and Ben Shen ${ }^{1,2,3,{ }^{*}}$ \\ 1 Division of Pharmaceutical Sciences, University of Wisconsin-Madison, Madison, Wisconsin 53705, USA \\ 2 University of Wisconsin National Cooperative Drug Discovery Group, University of Wisconsin-Madison, \\ Madison, Wisconsin 53705, USA \\ 3 Department of Chemistry, University of Wisconsin-Madison, Madison, Wisconsin 53705, USA
}

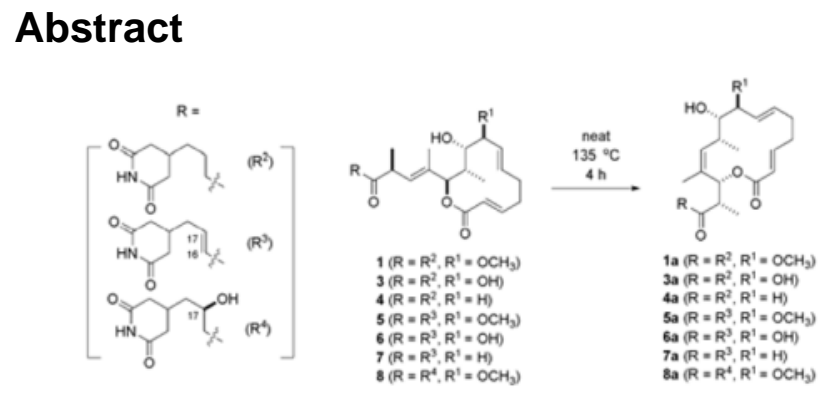

Thermolysis of iso-migrastatin (1) under neat heating conditions afforded migrastatin (1a). The reaction is proposed to proceed via a concerted $[3,3]$-sigmatropic rearrangement by which ring expansion is achieved regio- and enantio-specifically. The general applicability of this reaction was demonstrated with six additional iso-migrastatin congeners $(3-8)$, providing a new route to the synthesis of migrastatin analogs $(3 a-8 a)$.

\begin{abstract}
Migrastatin (1a), a 14-membered macrolide with a glutarimide-containing polyketide side chain, was first isolated by Imoto and co-workers from the culture broth of Streptomyces sp. MK929-43F1 in 2000. ${ }^{1}$ Its relative and absolute stereochemistry was subsequently determined by single-crystal X-ray analysis of its $\mathrm{N}-p$-bromophenacyl derivative in $2002{ }^{2}$ Dorrigocin A (2a) and B (2b), glutarimide-containing linear polyketides, were first isolated by Karwowski and co-workers from the culture broth of Streptomyces platensis NRRL 18993 in 1994, but the stereochemistry of $\mathbf{2 a}$ and $\mathbf{2 b}$ was not reported. ${ }^{3}$ Viewing $\mathbf{2 a}$ and $\mathbf{2} \mathbf{b}$ as acyclic geometric isomers of 1a, Licari and co-workers re-investigated the fermentation of S. platensis and confirmed in 2002 that this strain indeed produced $\mathbf{1 a}$, in addition to $\mathbf{2 a}$ and $\mathbf{2 b}$, as well as isomigrastatin (1), which could be viewed as the cyclic form of $\mathbf{2 b} .{ }^{4}$ The stereochemistry of $\mathbf{1}$ was also not reported. We have recently shown that $\mathbf{1 a}, \mathbf{2 a}, \mathbf{2} \mathbf{b}$, and $\mathbf{1}$ were produced by single biosynthetic machinery and 1a, 2a, 2b, as well as 13-epi-dorrigocin A (2c) are in fact shunt metabolites of $\mathbf{1}$ in $S$. platensis and subsequently established the relative and absolute stereochemistry of all these metabolites 5,6 (Figure 1).
\end{abstract}

bshen@pharmacy.wisc.edu. 
Migrastatin (1a) has shown the ability to inhibit the migration of several human tumor cell lines in vitro. ${ }^{7}$ Since the development of metastatic lesions remains the predominant cause of death for most cancer patients, and cell migration is essential for invasion of the extracellular matrix and for cell dissemination during metastasis, inhibition of tumor cell migration represents a potential therapeutic approach for the treatment of tumor metastasis. ${ }^{8}$ Thus, $\mathbf{1 a}$ thus has served as a potential lead for the development of therapeutic agents for treating tumor metastasis. Total synthesis of $\mathbf{1 a}$ has been achieved and a focused library of migrastatin analogs has been made by Danishefsky and co-workers. ${ }^{9}$ Strikingly, the macrolide core of $\mathbf{1 a}$ as well as its two analogs, macroketone and macrolactam, have been found to show improved biological profiles (by ca. 3 orders of magnitude) in inhibiting tumor cell migration in vitro and in vivo in comparison with 1a. ${ }^{10}$

Previously we have shown that $\mathbf{1}$ undergoes a rapid rearrangement in an aqueous solution to form $\mathbf{1 a}, \mathbf{2 a}, \mathbf{2 b}$, and $\mathbf{2} \mathbf{c}$ in quantitative yield. ${ }^{5}$ This finding has prompted us to further explore the chemical reactivity of $\mathbf{1}$. Here we report the thermolysis of $\mathbf{1}$ under neat heating conditions to afford 1a, presenting a biomimetic synthesis to $\mathbf{1 a}$ from $\mathbf{1}$ in a modest yield. We propose that the reaction would proceed via a concerted [3,3]-sigmatropic rearrangement by which ring expansion of the 12-membered macrolide 1 to the 14-membered macrolide 1a is achieved regio- and enantio-specifically. The general applicability of this reaction was demonstrated with six additional 1 congeners $(\mathbf{3}-\mathbf{8})$, providing a new route to the synthesis of $\mathbf{1}$ analogs $(3 \mathbf{a}-\mathbf{8 a})$.

We have reported early that $\mathbf{1}$ and its congeners such as $\mathbf{3}, \mathbf{4}, \mathbf{5}, \mathbf{6}, \mathbf{7}$, and $\mathbf{8}$, could be readily produced in substantial quantities by fermentation of Streptomyces platensis. 6 They were produced and isolated according to our reported procedures and utilized as starting materials for the synthesis of $1 \mathbf{a}$ and its analogues $3 \mathbf{a}, \mathbf{4 a}, 5 \mathbf{a}, \mathbf{6 a}, 7 \mathbf{a}$, and $8 \mathbf{a}$ in this study.

We started with 1 to optimize conditions for its thermal rearrangement to 1a. Numerous attempts to heat $\mathbf{1}$ in various solvents (such as toluene, DMF, DMSO, mineral oil) at temperatures ranging from $100{ }^{\circ} \mathrm{C}$ to $220^{\circ} \mathrm{C}$ were made initially. However, under all conditions tested, HPLC analysis of the reaction showed a complex mixture with the bulk of the starting material 1 decomposed or isomerized (data not shown). Surprisingly, when we resorted to thermolysis of neat $\mathbf{1}$ in a sealed glass tube, we observed the formation of $\mathbf{1 a}$ as the sole product as monitored by HPLC (Scheme 1).

We then optimized the reaction conditions by subjecting multiple aliquots of neat $\mathbf{1}(1 \mathrm{mg})$ in identical sealed tubes to thermolysis under various temperatures and duration, and the reactions were monitored by HPLC. The best yield was obtained when thermolysis of 1 was carried out at $135^{\circ} \mathrm{C}$ for $4 \mathrm{~h}$ under neat condition. We subsequently scaled up the thermolysis reaction of $\mathbf{1}(35 \mathrm{mg})$, and the resultant product $\mathbf{1 a}$ was purified by silica gel chromatography in a modest yield of $62 \%$. It should be pointed out that, even under the optimized reaction condition, 1 still suffered from decomposition or polymerization, which accounted for the modest yield obtained. The ${ }^{1} \mathrm{H}$ and ${ }^{13} \mathrm{C}$ NMR spectral data of the purified $1 \mathrm{a}$ were determined, and they are identical to those of $\mathbf{1 a}$ isolated directly from $\mathrm{S}$. platensis fermentation or obtained from $\mathrm{H}_{2} \mathrm{O}$ mediated rearrangement of $\mathbf{1}$, confirming its same stereochemistry.

We propose that the reaction would proceed through a concerted [3,3]-sigmatropic rearrangement, presumably via a chairlike transition state as depicted in Scheme 2. This proposal provides a plausible rationale for the observed absolute stereochemistry for $\mathbf{1 a}$ from 1. It is postulated that the $\mathrm{C}-14$ stereocenter, which possesses chiral bulk substituents, may induce asymmetry in the new $\mathrm{C}-\mathrm{O}$ bond formation, resulting in the $13 \mathrm{~S}$ configuration and $\mathrm{cis}$ C-11/C-12 double bond in the 14-membered product $\mathbf{1 a}$. 
We next subjected six additional congeners $(\mathbf{3}-\mathbf{8})$ of $\mathbf{1}$ to thermolysis to further investigate the generality of this reaction. Indeed, thermolysis of compounds $\mathbf{3}-\mathbf{8}$ under the optimized condition all yielded their corresponding rearranged products $\mathbf{3 a}-\mathbf{8 a}$ in acceptable yields ranging from $34 \%$ to $57 \%$ (Table 1 ).

Not surprisingly, compounds $3-\mathbf{7}$, which possess or lack an $8 \beta$-substitute and/or C-16/C-17 double bond, exhibited the same diastereofacial selectivity at C-13 of the resultant products $3 \mathbf{a}-7 \mathbf{a}$, each of which was produced essentially as a single diastereoisomer. Compound $\mathbf{8}$, bearing a 17-OH group, was the only exception that afforded two products, the expected product of 17-hydroxy-migrastatin $(\mathbf{8 a})$ and its further dehydrated product 16, 17-didehydromigrastatin (5a), in 3:1 ratio as judged by HPLC. The resultant products $\mathbf{3 a}-\mathbf{8 a}$ were each purified by silica gel chromography, and their ${ }^{1} \mathrm{H}$ and ${ }^{13} \mathrm{C}$ NMR spectra, as measured, were identical to those obtained via $\mathrm{H}_{2} \mathrm{O}$-mediated rearrangements, confirming that they have the same stereochemistry.

These examples support the general applicability of this reaction and illustrate that $\mathbf{1}$ analogs either possessing or lacking an $8 \beta$-substitute and a C-16/C-17 double bond is tolerable to this reaction, while 1 analog with 17-OH group is also tolerable but suffers from dehydration at high temperature. The presence of $8 \beta$-substituent in the $\mathbf{1}$ scaffold appears to favor the rearrangement to the 1a scaffold, while the presence of C-16/C-17 double bond in the 1 scaffold correlates to low yield for the desired product. Regardless of the substitution, the yields for this reaction remain modest $(34 \%-62 \%)$ mainly due to the decomposition of starting materials under the heat conditions.

Several other natural products, structurally related to $\mathbf{1}$, are known such as lactimidomycin (9) (Figure 1), which was first isolated by Sugawara and co-workers from Streptomyces amphibiosporus in $1992 .{ }^{11} \mathrm{We}$ isolated $\mathbf{9}$ and subjected it to thermolysis under identical conditions as optimized for $\mathbf{1}$ and its congeners. Surprisingly, no dinstinct products were observed, and $\mathbf{9}$ apparently underwent extensive decomposition or polymerization. This observation in fact is consistent with the proposed model: the presence of the C-8/C-9 double bond in 9 may decrease the flexibility of the 12-membered ring, hence making it difficult to form the transition state flavoring the desired [3,3]-sigmatropic rearrangement. Furthermore, the presence of the C-8/C-9 double bond enables 9 to possess a cis-diene system, which may be prone to react with other dienophiles intra- or intermolecularly, leading to polymerization.

In summary, we reported here that $\mathbf{1}$ can undergo thermolysis in neat condition to afford $\mathbf{1 a}$, and this reaction appears to proceed via a [3,3]-sigmatropic rearrangement by which ring expansion was achieved regio- and enantio-specifically. The general applicability of this reaction was demonstrated with six additional congeners of $\mathbf{1}$, providing a practical and efficient approach for the synthesis of a small set of $\mathbf{1 a}$ analogs. This strategy takes advantage of the unique reactivity of $\mathbf{1}$ and its congeners, which are readily available in large quantities by fermentation of $S$. platensis. Application of combinatorial biosynthesis methods to $\mathbf{1}$ biosynthesis in S. platensis promises the production of additional novel congeners of $\mathbf{1}$, thereby further expanding the library of 1a by the current methodology. It is hoped that an expanding library of 1a will greatly facilitate the development of therapeutic agents for treating tumor metastasis.

\section{Supplementary Material}

Refer to Web version on PubMed Central for supplementary material. 


\section{Acknowledgements}

We thank the Analytical Instrumentation Center of the School of Pharmacy, UW-Madison for support in obtaining MS and NMR data. This work is supported in part by NIH grants CA106150 and CA113297. B.S. is the recipient of an NIH Independent Scientist Award (AI51689).

\section{References}

1. Nakae K, Yoshimoto Y, Sawa T, Homma Y, Hamada M, Takeuchi T, Imoto M. J Antibiot 2000;53:1130-1136. [PubMed: 11132958]

2. (a) Nakae K, Yoshimoto Y, Ueda M, Sawa T, Takahashi Y, Naganawa H, Takeuchi T, Imoto M. J Antibiot 2000;53:1228-1230. [PubMed: 11132973] (b) Nakamura N, Takahashi Y, Naganawa H, Nakae K, Imoto M, Shiro M, Matsumura K, Watanabe H, Kitahara T. J Antibiot 2002;55:442-444. [PubMed: 12061554]

3. Hochlowski JE, Whittern DN, Hill P, McAlpine JB. J Antibiot 1994;47:875-880. [PubMed: 7928673]

4. Woo EJ, Starks CM, Carney JR, Arslanian R, Cadapan L, Zavala S, Licari P. J Antibiot 2002;55:141146. [PubMed: 12002995]

5. Ju J, Lim SK, Jiang H, Shen B. J Am Chem Soc 2005;127:1622-1623. [PubMed: 15700980]

6. Ju J, Lim SK, Jiang H, Seo JW, Shen B. J Am Chem Soc 2005;127:11930-11931. [PubMed: 16117518]

7. Takemoto Y, Nakae K, Kawatani M, Takahashi Y, Naganawa H, Imoto M. J Antibiot 2001;54:11041107. [PubMed: 11858668]

8. Woodhouse EC, Chuaqui R, Liotta LA. Cancer 1997;80:1529-1537. [PubMed: 9362419]

9. Gaul C, Njardarson JT, Shan D, Dorn DC, Wu KD, Tong WP, Huang XY, Moore MA, Danishefsky SJ. J Am Chem Soc 2004;126:11326-11337. [PubMed: 15355116] and references cited therein

10. Shan D, Chen L, Njardarson JT, Gaul C, Ma X, Danishefsky SJ, Huang XY. Proc Natl Acad Sci USA 2005;102:3772-3776. [PubMed: 15728385]

11. Sugawara K, Nishiyama Y, Toda S, Komiyama N, Hatori M, Moriyama T, Sawada Y, Kamei H, Konishi M, Oki T. J Antibiot 1992;45:1433-1441. [PubMed: 1429229] 


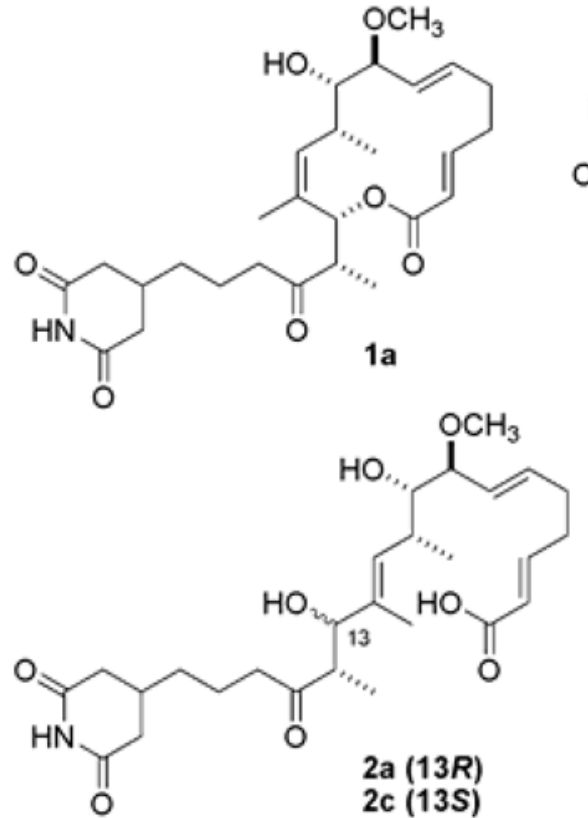<smiles>CO[C@H](/C=C/CC/C=C/C(=O)O)[C@@H](O)[C@@H](C)[C@@H](O)/C(C)=C/[C@@H](C)C(=O)CCCC1CC(=O)NC(=O)C1</smiles>

2b<smiles>CO[C@H](C)[C@@H](/C=C/CC/C=C/C(=O)OC(/C(C)=C/[C@@H](C)C(=O)CCCC1CC(=O)NC(=O)C1)[C@@H](C)O)OC</smiles><smiles>C/C=C\CC/C=C\C=C/C(=O)O[C@@H](C)/C(C)=C/[C@@H](C)C(=O)CC(O)CC1CC(=O)NC(=O)C1</smiles>

Figure 1.

Structures of migrastatin (1a), dorrigocin A (2a), dorrigocin B (2b), 13-epi-dorrigocin A (2c), iso-migrastatin (1), and lactimidomycin (9). 
<smiles>CCCCCC1CC(=O)NC(=O)C1</smiles><smiles>[R]C(=O)C(C)C=C(C)C(OC(=O)C=CCCC=CC(C)OC)[C@@H](C)[C@@H](C)O</smiles><smiles>[R]C(=O)[C@H](C)[C@@H](OC(=O)/C=C\CC/C=C/[C@@H](OC)[C@@H](O)[C@@H](C)C=C(C)C)[C@H](C)O</smiles>

1

$1 a$

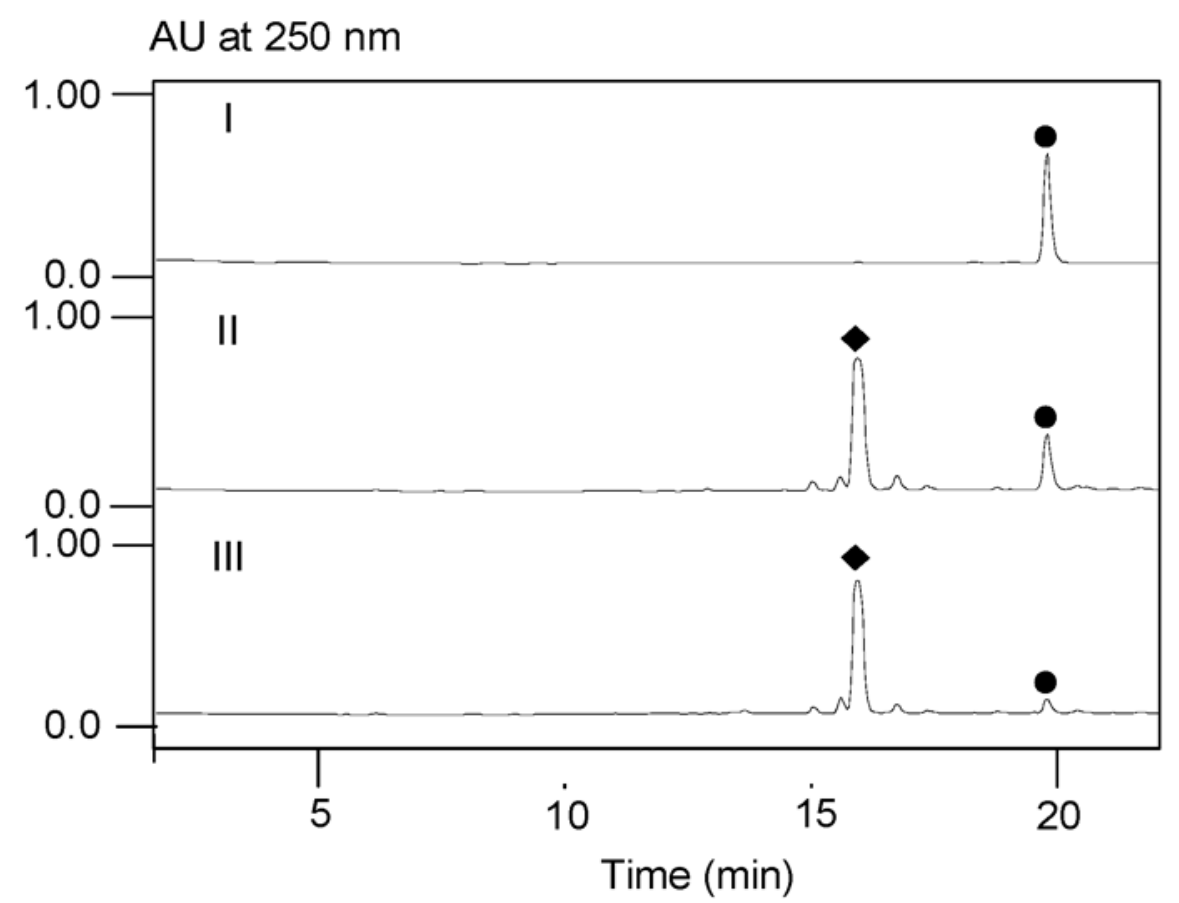

Scheme 1.

Thermolysis of iso-migrastatin (1) to afford migrastatin (1a): HPLC analysis of $\mathbf{1}(\bullet)$ as an substrate (I) and $\mathbf{1 a}(>)$ as a product upon heating $\mathbf{1}$ in a sealed glass tube $135^{\circ} \mathrm{C}$ for $2.5 \mathrm{hrs}$ (II) and (III) for $4 \mathrm{hrs.}$ 

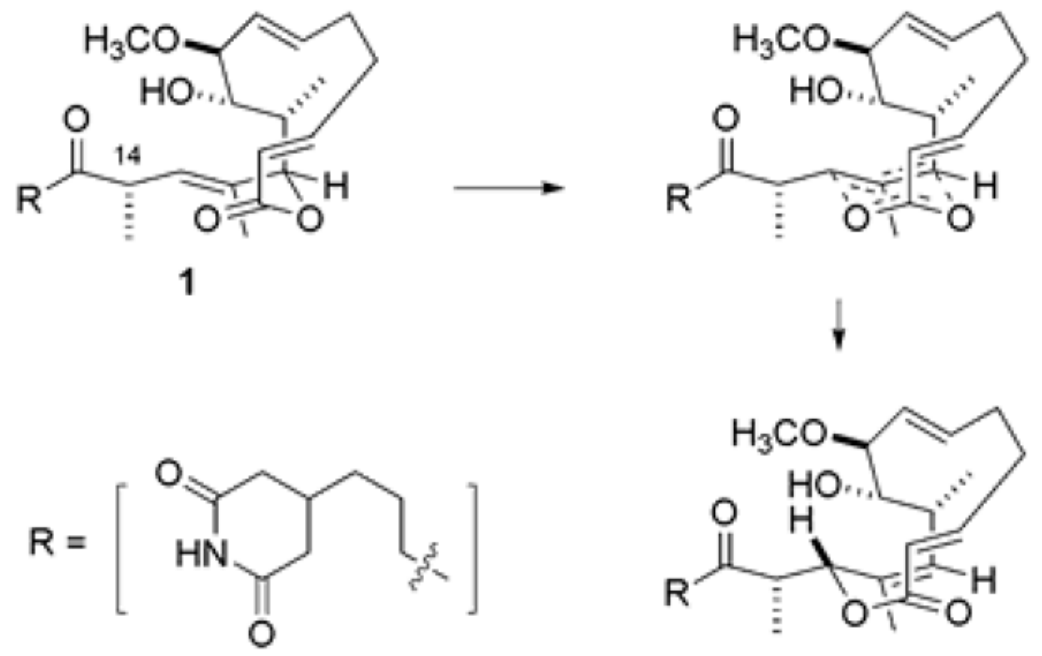

1a

Scheme 2.

Proposed mechanism of [3,3]-sigmatropic rearrangement of iso-migrastatin (1) to migrastatin (1a). 
Table 1

Thermolysis of iso-migrastatin $(\mathbf{1})$ and congeners $(\mathbf{3}-\mathbf{8})$ to migrastation $(\mathbf{1 a})$ and congeners $(\mathbf{3 a}-\mathbf{8 a})$ via a [3,3]sigmatropic rearrangement

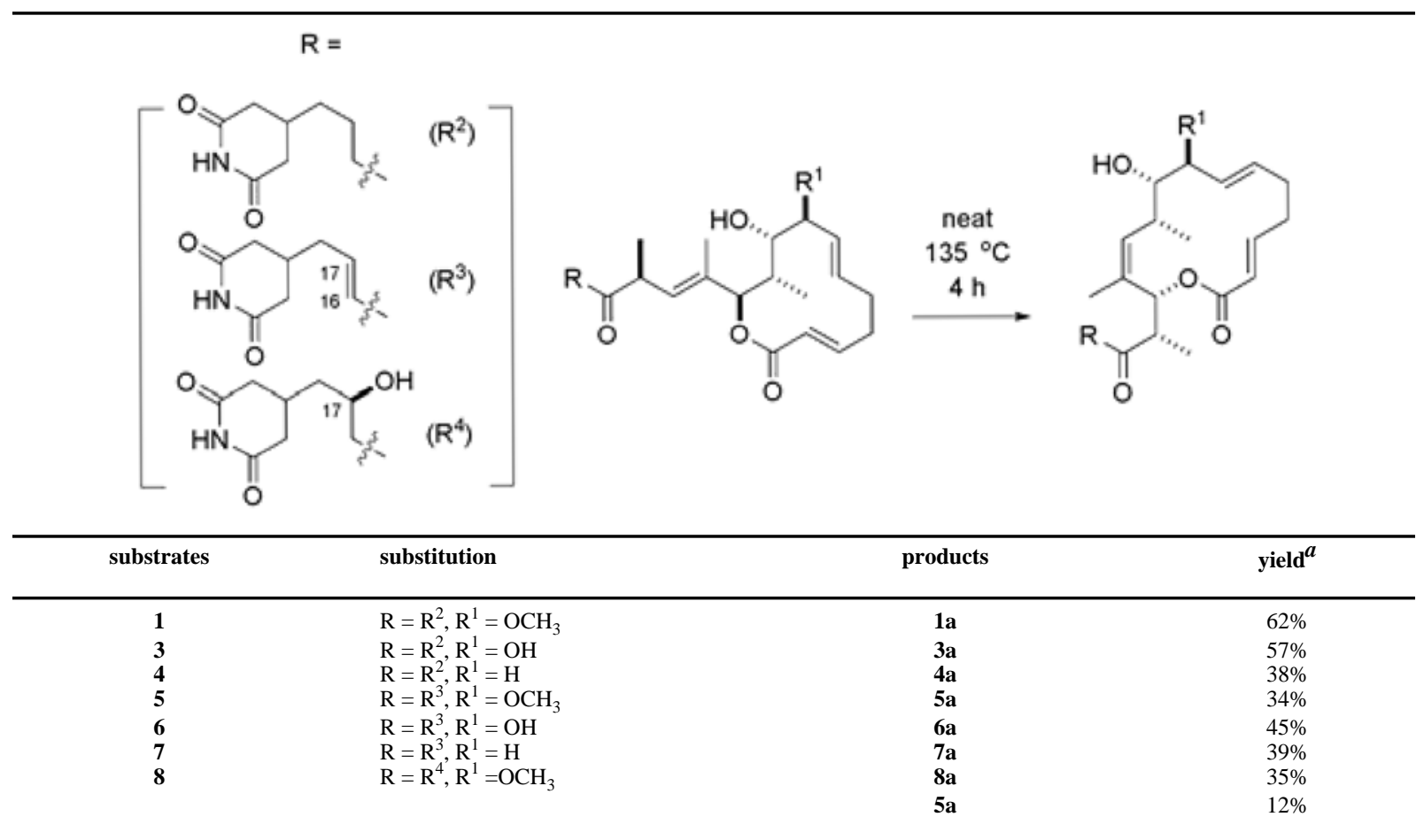

$a_{\text {Isolated yield }}$ 